\title{
Attenuated Vector Tomography - An Approach to Image Flow Vector Fields with Doppler Ultrasonic Imaging
}

\author{
Qiu Huang, Qiyu Peng, Bin Huang, Arvi Cheryauka, and \\ Grant T. Gullberg
}

E. O. Lawrence Berkeley National Laboratory,

Life Science Division

Berkeley, CA

This work was supported in part by the Director, Office of Science, Office of Biological and Environmental Research, Medical Sciences Division of the U.S. Department of Energy under Contract DE-AC02-05CH11231.

Qiu Huang (e-mail: qhuang@lbl.gov), Qiyu Peng (e-mail: QPeng@lbl.gov), Bin Huang (e-mail: BHuang@lbl.gov) and Grant T. Gullberg (e-mail: gtgullberg@lbl.gov) are with Lawrence Berkeley National Laboratory, One Cyclotron Road, MS 55R0121, Berkeley, CA 94720-8119.

Arvi Cheryauka is with the GE Healthcare, Salt Lake City, UT (e-mail: Arvi.Cheryauka@med.ge.com). 
Abstract - The measurement of flow obtained using continuous wave Doppler ultrasound is formulated as a directional projection of a flow vector field. When a continuous ultrasound wave bounces against a flowing particle, a signal is backscattered. This signal obtains a Doppler frequency shift proportional to the speed of the particle along the ultrasound beam. This occurs for each particle along the beam, giving rise to a Doppler velocity spectrum. The first moment of the spectrum provides the directional projection of the flow along the ultrasound beam. Signals reflected from points further away from the detector will have lower amplitude than signals reflected from points closer to the detector. The effect is very much akin to that modeled by the attenuated Radon transform in emission computed tomography.

A least-squares method was adopted to reconstruct a 2D vector field from directional projection measurements. Attenuated projections of only the longitudinal projections of the vector field were simulated. The components of the vector field were reconstructed using the gradient algorithm to minimize a least-squares criterion. This result was compared with the reconstruction of longitudinal projections of the vector field without attenuation. If attenuation is known, the algorithm was able to accurately reconstruct both components of the full vector field from only one set of directional projection measurements. A better reconstruction was obtained with attenuation than without attenuation implying that attenuation provides important information for the reconstruction of flow vector fields.

This confirms previous work where we showed that knowledge of the attenuation distribution helps in the reconstruction of MRI diffusion tensor fields from fewer than the required measurements. In the application of ultrasound the attenuation distribution is obtained with pulse wave transmission computed tomography and flow information is obtained with continuous wave Doppler. 


\section{INTRODUCTION}

The Doppler flow measurements obtained using a directional beam of a continuous ultrasound wave can be formulated as a directional projection of a flow vector field [1,2,3]. The continuous wave reflects from points at different distances from the ultrasound detector. Signals reflected from points further away from the detector will have lower amplitude than signals reflected from points closer to the detector. This effect can be thought of as being very much akin to the attenuated Radon transform in emission computed tomography. Previous work performed by our group [4] indicates that when there is no attenuation, the solenoidal component of the vector field can be reconstructed from the longitudinal projection of the vector field and the irrotational component can be reconstructed from the transversal projection. When there is attenuation, however, the full twodimensional (2D) vector field can be reconstructed from one directional projection data set [5-8]. In this paper, we investigate whether introducing a known attenuator to the directional projection measurements helps reconstruction of a vector field from only the longitudinal measurements and thus, exploits the potential use of attenuation in reducing the required number of measurements.

\section{Modeling Doppler Measurements}

When a continuous ultrasound beam bounces against a flowing particle, a signal is backscattered. This signal obtains a Doppler frequency shift proportional to the speed of the particle along the beam. This occurs for each particle along the beam giving rise to a Doppler velocity spectrum. The first moment of the spectrum provides the directional projection of the flow along the ultrasound beam.

The following development follows that presented in [1,2]. Consider a stationary flow vector field $f$ in a vessel. At two adjacent points outside the body, a transmitter and a receiver are placed. Assume that a collimated ultrasonic wave $\boldsymbol{a}(\boldsymbol{t})=\boldsymbol{e}^{i \boldsymbol{\omega}_{0} t}$ of frequency $\boldsymbol{\omega}_{0}$ is transmitted along an oriented straight line $\boldsymbol{L}$ with velocity $\boldsymbol{c}$ and meets a particle with velocity $\boldsymbol{v}$ along $\boldsymbol{L}$ ( $\boldsymbol{v}$ is the projected component of $\boldsymbol{f}$ onto $\boldsymbol{L}$ ), then the reflected signal obtains a Doppler frequency shift

$$
\delta_{\omega}=-\frac{2 c \omega_{0} v}{c^{2}-v^{2}}
$$

If $|v|<<c$ then the approximate frequency shift is

$$
\boldsymbol{\delta}_{\boldsymbol{\omega}}=\boldsymbol{k} \boldsymbol{v} \text { with } \boldsymbol{k}=-\frac{2 \boldsymbol{\omega}_{0}}{\boldsymbol{c}} .
$$

If the reflection of the signal can be modeled by a reflectivity coefficient $\boldsymbol{\rho}$, then the signal after reflection at the point $\mathrm{x}$ can be written as

$$
b_{x}(t)=\rho(x) e^{i\left(\omega_{0}+k v\right) t}
$$

For a stream of particles, the superposition of the contributions from each particle gives rise to the reflected signal

$$
b(t)=\int_{\Re} e^{i\left(\omega_{0}+k v\right) t} \sigma(f, \rho, L, v) d v,
$$

where $\boldsymbol{\sigma}(\boldsymbol{f}, \boldsymbol{\rho}, \boldsymbol{L}, \boldsymbol{v}) \boldsymbol{d} \boldsymbol{v}$ is a weight depending on the reflectivity $\boldsymbol{\rho}$ and on the velocity component along $\boldsymbol{L}$ of the reflecting particles. More precisely, if $\boldsymbol{e}_{\boldsymbol{L}}$ is the unit directional vector of $\boldsymbol{L}$ and $\boldsymbol{d} \boldsymbol{s}$ is the Lebesgue measure on $\mathfrak{R}$, then

$$
\sigma(f, \rho, L, v) d v=\underset{\left\{x \in L \mid v \leq e_{L} \cdot f(x)<v+d v\right\}}{\int \rho(x) d s}
$$

$\boldsymbol{\sigma}(\boldsymbol{f}, \boldsymbol{\rho}, \boldsymbol{L}, \boldsymbol{v})$ can be thought of as the velocity spectrum of $f$ along $L$. The first moment of $\sigma(f, \rho, L, v) d v$ is

$$
\int_{L} v \sigma(f, \rho, L, v) d v=\int_{L} f \cdot d S_{L}
$$

If $\boldsymbol{d} \boldsymbol{S}_{\boldsymbol{L}}=\boldsymbol{e}_{\boldsymbol{L}} \boldsymbol{d s}$, then the previous expression provides the Doppler-Radon transform

$$
(f, L) \rightarrow \int_{L} v \sigma(f, \rho, L, v) d v=\int_{L} f \cdot d S_{L}=\int_{L} f \cdot e_{L} d s
$$

Now let us write $\boldsymbol{\sigma}(\boldsymbol{f}, \boldsymbol{\rho}, \boldsymbol{L}, \boldsymbol{v})$ in terms of the signal $\boldsymbol{b}(\boldsymbol{t})$

$$
\int_{\Re} e^{i \tau v} \sigma(f, \rho, L, v) d v=e(\tau)=b(\tau / k) e^{-i \omega_{0} \tau / k}
$$

Therefore,

$$
\boldsymbol{\sigma}(\boldsymbol{f}, \boldsymbol{\rho}, \boldsymbol{L}, \boldsymbol{v})=\mathfrak{I}[\boldsymbol{e}(\tau)]=\mathfrak{I}\left[\boldsymbol{b}(\tau / \boldsymbol{k}) \boldsymbol{e}^{-i \omega_{0} \tau / k}\right]
$$

We see that sigma is a rescaling of the Fourier transform of the signal around $\boldsymbol{\omega}_{0}$. From this we can write the Radon-Doppler transform

$$
(f, L) \rightarrow \int_{L} v \mathfrak{I}\left[b(\tau / k) e^{-i \omega_{0} \tau / k}\right] d v=\int_{L} f \cdot e_{L} d s
$$

\section{VECTOR TOMOGRAPHY}

In this section we will define the vector tomography problem as scalar measurements given by the integral transforms of the vector field

$$
(\boldsymbol{P V})_{\overline{\boldsymbol{\omega}}}(\boldsymbol{\theta}, \boldsymbol{s})=\int_{L: \vec{x} \cdot \vec{\theta}=s} \overrightarrow{\boldsymbol{\omega}} \cdot \vec{V}(\overrightarrow{\boldsymbol{x}}) d \overrightarrow{\boldsymbol{x}},
$$

where $\boldsymbol{L}$ is some line in space and $\overrightarrow{\boldsymbol{\omega}}$ is a unit vector indicating some direction. The attenuated Radon transform of the vector field is given by

$$
\left(\boldsymbol{P}_{\mu} \boldsymbol{V}\right)_{\overline{\boldsymbol{\omega}}}(\boldsymbol{\theta}, \boldsymbol{s})=\int_{L: \vec{x} \cdot \overrightarrow{\boldsymbol{\theta}}=s} \overrightarrow{\boldsymbol{\omega}} \cdot \vec{V}(\overrightarrow{\boldsymbol{x}}) e^{-\int_{0}^{\infty} \boldsymbol{\mu}\left(\overrightarrow{\boldsymbol{x}}+t \overline{\boldsymbol{\theta}}^{\perp}\right) d t} d \overrightarrow{\boldsymbol{x}}
$$

In $2 \mathrm{D}$ vector tomography, two typical transforms are the longitudinal transform, where $\overrightarrow{\boldsymbol{\omega}}$ is along the line $\boldsymbol{L}$ of integration, and the transversal transform where $\overrightarrow{\boldsymbol{\omega}}$ is orthogonal to $\boldsymbol{L}$. It is known that both the longitudinal and the transversal projections are necessary to reconstruct the vector field $\vec{V}$. However, if these transforms are attenuated, only one of them is necessary to reconstruct the full vector field [5]-[8]. An analytical algorithm is presented in [5] and [6] for reconstructing the complete vector field from attenuated longitudinal projections; 
and an analytical algorithm is presented in [7] for reconstructing the complete field from attenuated transversal projections. Without attenuation, the reconstruction of the longitudinal projections provides the solenoidal component and reconstruction of the transversal projections provides the irrotational component of the vector field.

\section{NUMERICAL RESULTS}

In this paper a least squares method was used in the reconstruction of the full vector field $\vec{V}(x)$ from simulated projection measurements with and without attenuation. The objective function was chosen to be

$$
\mathrm{X}^{2}(\overrightarrow{\boldsymbol{V}})=\sum_{\boldsymbol{\theta}, \boldsymbol{s}} \sum_{\overline{\boldsymbol{\omega}}}\left[\left(\boldsymbol{P}_{\boldsymbol{\mu}} \boldsymbol{V}\right)(\boldsymbol{\theta}, \boldsymbol{s})-\boldsymbol{g}_{\overline{\boldsymbol{\omega}}}(\boldsymbol{\theta}, \boldsymbol{s})\right]^{2},
$$

where $\boldsymbol{g}_{\overrightarrow{\boldsymbol{\omega}}}$ are the directional projection measurements, and the summation is for all projection samples at detector positions $s$ and angle $\boldsymbol{\theta}$ and all directional measurements $\overrightarrow{\boldsymbol{\omega}}$. The reconstruction results were obtained using the gradient algorithm. In this simulation, directional projections were used where the unit vector $\overrightarrow{\boldsymbol{\omega}}$ was along the direction of the projections. In Fig. 1 , the reconstruction of the vector components is shown. The reconstruction (last column) from non attenuated projections is neither a pure solenoidal component of the vector field nor a pure irrotational component. The components of the vector field seem to bleed into the other component producing ghosts in the other component. The best results are obtained with the reconstruction of attenuated longitudinal directional projections with knowledge of the attenuation.
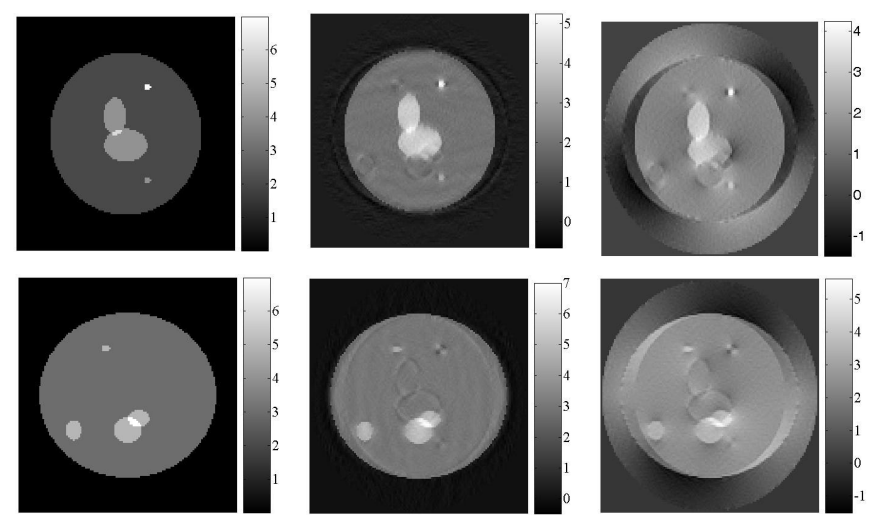

Figure 1. Vector Field Reconstructions From Longitudinal Projection Measurements. The top and bottom rows are the first and second component of the 2-D vector field. The first column is the original, the second column is the reconstruction of attenuated projections, and the last column is the reconstruction without attenuation.

\section{DISCUSSION}

In this work we simulated the reconstruction of vector fields from their scalar projections, both with and without attenuation. The comparisons show that when using a simple least squares criterion, attenuation helps in reconstructing the full vector field from insufficient measurements. We show that this has application for Doppler ultrasonic imaging, but may have implication for diffusion tensor MR tomographic imaging.

\section{REFERENCES}

[1] G. Sparr and K. Strahlen, "Vector field tomography, an overview," in IMA Volumes in Mathematics and its Applications; Computational Radiology and Imaging: Therapy and Diagnostic, vol 110, Springer Verlag.

[2] G. Sparr, K. Strahlen, K. Lindstrom, and H. W. Persson, "Doppler tomography for vector fields," Inverse Problems, vol. 11, pp. 1051-1061, 1995.

[3] K. Strahlen, "Exponential vector field tomography," in Lecture Notes in Computer Science, 1311: Image analysis and Processing. A. Dei Bimbo (ed), Springer, IAPR, 1997, 348-355.

[4] M. Defrise and G.T. Gullberg, " $3 D$ Reconstruction of Tensors and Vectors," Tech. Rep. LBNL-54936, 2005.

[5] A.A. Bukhgeim and S.G. Kazantsev, "Inversion formula for the fan-beam attenuated Radon transform in a unit disk," Russian Academy of Science Siberian Branch: The Sobolev Institute of Mathematics, Preprint No.99, 2002.

[6] A.A. Bukhgeim and S.G. Kazantsev, "Full reconstruction of a vector field from its attenuated vectorial Radon transform," in Proceedings of the 22nd IASTED International Conference MODELING, IDENTIFICATION, AND CONTROL, pp. 294-298, 2003.

[7] F. Natterer, "Inverting the attenuated vectorial Radon transform," J. Inv. IllPosed Problems, vol 13, pp. 93-101, 2005.

[8] Q. Huang and G. T. Gullberg, "Attenuation corrected tensor tomography Attenuation helps in the case of insufficient measurements," in Conference Record of the 2007 IEEE Nuclear Science Symposium and Medical Imaging Conference, Honolulu, Hawaii, 2007, vol. 6, pp. 4103-4109. 